\title{
Elaboración y aplicación de un índice de calidad ambiental para la región del nordeste argentino, 2010
}

\section{Development and implementation of an environmental quality index for the northeastern Argentine region, 2010}

\author{
Juan Pablo Celemín \\ Guillermo Ángel Velázquez*
}

\begin{abstract}
Argentina lacks environmental indices at national scale. To begin to fill this gap, this paper presents an environmental quality index for the departments comprised in the northeastern provinces of Argentina. It is composed of twenty-three variables grouped into three dimensions known as: a) naturebased recreation resources; b) socially constructed resources; and, c) environmental problems. A proper consideration of environmental inequality provides elements to formulate appropriate policies to mitigate these problems.
\end{abstract}

Keywords: environmental quality index, Northeastern Region (Argentina), environmental quality.

\section{Resumen}

Argentina no cuenta con índices ambientales a escala nacional, para suplir esta carencia la presente investigación plantea la elaboración de un Índice de Calidad Ambiental (ICA) para los departamentos que constituyen las provincias del nordeste del país que contiene veintitrés variables agrupadas en tres grandes dimensiones denominadas $a$ ) recursos recreativos de base natural; $b$ ) recursos socialmente construidos y c) problemas ambientales que fueron valoradas a partir de la conjunción de puntuaciones subjetivas y objetivas. Una adecuada y completa aproximación al tema ambiental brinda elementos útiles para formular políticas de intervención que mitiguen las asimetrías socioterritoriales.

Palabras clave: Índice de Calidad Ambiental, región nordeste (Argentina), calidad ambiental.

* Consejo Nacional de Investigaciones Científicas y Técnicas. Universidad Nacional del Centro de la Provincia de Buenos Aires, Argentina. Correos-e: jpcelemin@conicet.gov.ar,gvelaz@fch.unicen. edu.ar 


\section{Introducción}

Los índices sirven para informar al público general y especialmente a los agentes con capacidad de decisión acerca de las estrategias necesarias para mejorar el bienestar de la población. La importancia actual de los índices ambientales yace en la creciente demanda de mayor y mejor información ambiental, en particular por algunos sectores sociales con creciente interés por los aspectos ambientales del desarrollo y el bienestar social. A medida que mejora su situación socioeconómica surgen nuevos intereses y preocupaciones, tal es el caso del ambiente y la ecología.

La mayor limitante en la elaboración de estos índices es la accesibilidad, disponibilidad y confiabilidad de los datos, principalmente aquellos presentados con mayor nivel de desagregación. Más aún la agregación y simplificación de la información, con el objetivo de la divulgación científica, reduce el poder analítico de los resultados pero, a su vez, hace visibles a todos los estratos de la sociedad gran cantidad de datos resumidos.

Diferentes organismos nacionales, provinciales y municipales elaboran periódicamente indicadores para conocer el contexto socioeconómico de sus correspondientes jurisdicciones, las variables ambientales poseen escalas de análisis espaciales y temporales diferenciales. Esta característica obstaculiza el trabajo de concordar la información ambiental con la socioeconómica. Por tanto, un análisis como el presente debe buscar un compromiso entre escala de análisis, disponibilidad de información y recursos existentes para poder cumplimentar sus objetivos.

En este contexto, el objetivo de esta investigación es elaborar y mostrar la distribución espacial de un Índice de Calidad Ambiental (ICA) que se instaure en los departamentos que conforman el noreste de la República Argentina, considerando los recursos recreativos (tanto de base natural como socialmente construidos) y los costos ambientales, esto, sin dejar de reconocer sus disparidades en el territorio en cuestión. Consiguientemente, el ICA se propone, por un lado, dimensionar la magnitud de algunos problemas ambientales que afectan el bienestar de la población y, por otra parte, una cuantificación de la percepción en el caso de elementos subjetivos como la valoración de los recursos escénicos o de los elementos de esparcimiento.

El índice resalta la utilidad de planificar la intervención del territorio no sólo en función de las condiciones socioeconómicas, sino también a partir de la consideración de variables ambientales disponibles en la misma escala y, por tanto, compatibles con las variables socioeconómicas. Entonces se puede sugerir realmente una planificación "integral" del territorio y no una parcial que considere un amplio espectro de los fenómenos que se presentan en el espacio cotidiano de los individuos. 


\section{La compleja estructura de un índice ambiental}

La elaboración y seguimiento del índice puede realizarse bajo los enfoques subjetivo y objetivo. Mientras que el primero se basa en información de origen secundario, el último considera a las percepciones que proporcionan una visión más completa que las observaciones frías y "objetivas" proporcionadas por los datos. Siempre que se utilicen adecuadamente estos enfoques y se comprendan los alcances y limitaciones de cada uno pueden ser utilizados complementariamente (Sterimberg, et al., 2004).

Un índice puede integrarse por indicadores ambientales cualitativos y cuantitativos, pero la mayoría de las definiciones de éstos excluye el uso de variables cualitativas. Los indicadores describen los fenómenos complejos de una forma (casi) cuantitativa mediante la simplificación de la información de tal manera que es posible la comunicación con grupos de usuarios específicos. El término "casi" indica que, aunque los indicadores son en su mayoría de naturaleza cuantitativa, en principio, también pueden ser de tipo cualitativo (Rotmans, 1997). Por su parte, estos últimos pueden ser preferibles a los indicadores cuantitativos cuando la información cuantitativa no está disponible o el tema de interés no es inherentemente cuantificable (Gallopin, 1996; 2006) y aunque estén basados en la observación deben ser estandarizados a una forma numérica para hacerlos comparables y compatibles con las variables cuantitativas (Pieri, et al., 1995).

El uso de enfoques subjetivos ya está presente en el campo del medio ambiente, pues es una práctica muy común en la Evaluación de Impacto Ambiental (EIA), basado principalmente en la experiencia profesional. Las valoraciones de los expertos que participan en una Evaluación de Impacto Ambiental desempeñan un papel significativo en sus resultados debido a la considerable subjetividad en la toma de decisiones sobre la cual se basa la EIA (Wilkins, 2003). Podemos decir, entonces, que hay algún paralelismo entre esta afirmación y nuestra propuesta de selección y la medición de variables en las que, al igual que en el EIA, se reconocen juicio profesional, la experiencia, la intuición y el valor (Weston, 2000).

El Índice de Calidad Ambiental propuesto considera no sólo a las habituales variables vinculadas con el medio físico-natural, sino también al espacio construido ya que es en él donde la población desarrolla su cotidianidad. Como afirma Santos (1996) es aquí donde la geografía puede brindar una contribución importante para otras disciplinas, porque lo "cotidiano" pasa a ser definido por el lugar, esto es, la manera como la gente vive habitualmente y se vincula territorialmente en una ciudad determinada.

Por tanto, se hace necesario recurrir a una concepción amplia del ambiente, es decir, el conjunto de las diferentes relaciones establecidas 
entre la sociedad y el medio físico, construido o hecho artificialmente, que tiene lugar en un espacio territorial acotado. Implica considerar simultáneamente usos de la tierra yuxtapuestos entre sí, multiplicidad de procesos y actores productores y reproductores de ese medio, variedad de significados y símbolos culturales (Herzer y Gurevich, 1996) que alcanzan su máxima expresión en las ciudades y su entorno, ya que producen un ambiente que les es propio, cuya principal característica es estar "socialmente construido", lo que lo diferencia fundamentalmente del medio natural estudiado por la ecología (Metzger, 2006).

Consiguientemente, el índice también incluye las variables asociadas con la calidad de vida desde una perspectiva más amplia como pueden ser la seguridad y las amenidades urbanas, a la vez que intenta diferenciarse de los índices elaborados por economistas que recurren a métodos hedónicos o de valoración contingente. ${ }^{1}$ La crítica a ese tipo de procedimientos es su reduccionismo economicista dado que todos los indicadores considerados tienen que estar asociados con el valor monetario. Rogerson (1999) y McCann (2004) los cuestionan ya que de esta manera la ciudad o el municipio son vistos exclusivamente como bienes que son parte de un proceso de competición interurbana por la atracción del capital y de recursos humanos calificados.

En tal sentido, Araña et al. (2003) sostiene que el territorio debe ser gestionado desde organismos públicos que no tengan en cuenta únicamente al mercado como elemento de asignación de los bienes ambientales.

\section{Los conceptos de calidad e inequidad ambiental}

Calidad ambiental es un concepto ambiguo tratado por multiplicidad de disciplinas que dificultan establecer una definición precisa de lo que se entiende por ella. Las producciones científicas, en general, la tratan de manera implícita y el lector debe analizar a los indicadores utilizados para determinar a qué tipo de definición se aproxima. Suele asociarse a otra clase de conceptos -igualmente de difusos y complejos- tales como calidad de vida, sostenibilidad y habitabilidad (Van Kamp et al., 2003).

\footnotetext{
${ }^{1}$ El primer modelo desglosa el precio de un bien privado, de mercado, en función de varias características, las cuales tienen un precio implícito cuya suma determina, en una proporción estimable, el precio del bien de mercado que se observa. Un ejemplo clásico del uso del modelo de los precios hedónicos es el de la externalidad negativa que producen los aviones sobre los residentes próximos a aeropuertos. El segundo es una técnica para estimar el valor de bienes (productos o servicios) para los que no existe mercado. Es extraordinariamente simple en su comprensión intuitiva: se trata de simular un mercado mediante encuestas a los consumidores potenciales. Se les pregunta por la máxima cantidad de dinero que pagarían por el bien si tuvieran que compararlo, como hacen con los demás bienes (Riera, 1994).
} 
Dada la versatilidad de dicha noción, Escobar (2006) indica que la calidad ambiental puede ser concebida como un componente más del desarrollo sostenible urbano, junto con las condiciones económicas y sociales. Un aporte abarcativo es el de Luengo (1998) que entiende por calidad ambiental a las condiciones óptimas que rigen al comportamiento del espacio habitable en términos de confort asociados a lo ecológico, biológico, económico-productivo, sociocultural, tipológico, tecnológico y estético en sus dimensiones espaciales. Así, la calidad ambiental es, por extensión, producto de la interacción de estas variables para la conformación de un hábitat saludable, confortable y capaz de satisfacer los requerimientos básicos de sostenibilidad de la vida humana individual y en interacción social dentro del medio urbano.

Por su parte, la inequidad ambiental sostiene que los sectores sociales con menores recursos son los que están frecuentemente más expuestos y afectados por los riesgos ambientales (contaminación del aire, mala calidad de las viviendas, calles sucias, alto tránsito vehicular y pocas comodidades a nivel local). También considera que es el sector de la población que tiene menor acceso a los bienes ambientales (energía suficiente, alimentos saludables y agua limpia), circunstancia que tiene efectos negativos importantes en la salud y en el bienestar de las personas (Catalán-Vázquez y Jarillo-Soto, 2010).

Por tanto refiere a la situación en la cual un grupo social específico es afectado significativamente por los riesgos ambientales, a diferencia de la justicia ambiental -con la cual está estrechamente asociada- que sostiene el trato justo y la participación significativa de todas las personas y comunidades en el desarrollo, implementación y aplicación de las políticas, leyes y regulaciones ambientales (Brulle y Pellow, 2006). Comparte con la noción de calidad ambiental el hecho de ser estudiada por diferentes disciplinas (demografía, sociología, economía, entre otras) pero es la ciencia geográfica la que puede diferenciarse a partir de la aplicación del análisis espacial de la inequidad ambiental, en particular a través del uso de los Sistemas de Información Geográfica (Szas y Meuser, 1997).

\section{La región del nordeste argentino (NEA): breve caracterización}

Entre el mosaico de regiones que conforman la República Argentina, la del NEA (mapa I), es la región más atrasada y desposeída del país, el cual está constituido por 23 provincias y la Ciudad Autónoma de Buenos Aires. A su vez, las provincias se subdividen en departamentos, que es la unidad de análisis requerida para este estudio. Las provincias también pueden agruparse de acuerdo a sus similitudes físico-naturales y socioeconómicas 
en regiones. No obstante este tipo de agrupamiento no conforma ningún orden administrativo, a diferencia de las provincias y departamentos.

En términos generales, para el Instituto Nacional de Estadísticas y Censos (2003), la región del NEA abarca íntegramente las provincias de Chaco, Formosa, Corrientes y Misiones. La tabla 1 muestra la cantidad de departamentos, población y superficie, mientras que el mapa I exhibe la ubicación de las provincias de la región en cuestión dentro de la República Argentina.

\section{Tabla 1}

Cantidad de departamentos, población y superficie de las provincias de la región del NEA

\begin{tabular}{lccc}
\hline \multicolumn{1}{c}{ Provincia } & Núm. departamentos & Población (hab.) & Superficie $\left(\mathrm{km}^{2}\right)$ \\
\hline Formosa & 9 & 527.895 & 72.066 \\
Chaco & 25 & 1.053 .466 & 99.633 \\
Misiones & 17 & 1.101 .593 & 29.801 \\
Corrientes & 25 & 992.595 & 88.199 \\
Total & 76 & 3.675 .549 & 289.699 \\
\hline
\end{tabular}

Fuente: elaboración personal.

El NEA es, tal como lo definieron Bruniard y Bolsi (1992), un variado mosaico de paisajes geográficos por asociación de diversas formas naturales y formas culturales.

Meichtry y Fantín (2001) sostienen que ni siquiera en la actualidad la generalización de las condiciones de deterioro social y económico permiten su reconocimiento como una unidad regional. De acuerdo con lo planteado por las autoras, podemos afirmar que la región asentada sobre formaciones del antiguo macizo de Brasilia, de este a oeste, poseen diferencias fisiográficas importantes, determinadas por historias geológicas y sistemas morfoclimáticos diferentes. Sobre éstas, el devenir histórico, con sus diferentes procesos de poblamiento y de desarrollo socioeconómico, contribuyó a la siguiente diferenciación espacial: ${ }^{2}$

1) La subregión misionera se caracteriza por sus formaciones de basaltos, diabasas, areniscas y cuarcitas (Popolizio, 1963). Estas son puestas en evidencia por la erosión fluvial que genera atractivas rupturas de pendiente en un marco de frondosa vegetación. Sus suelos rojos y sus condiciones hídricas favorables han contribuido a sostener una densa selva hidrófila, adaptada a zonas con abundante presencia de agua, que fue la

\footnotetext{
${ }^{2}$ Para mayores detalles sobre estas subregiones recomendamos los trabajos de Meichtry y Fantín (2001) y Meichtry y Fantín (2008).
} 


\section{Mapa I \\ Ubicación de la región nordeste de la República Argentina}

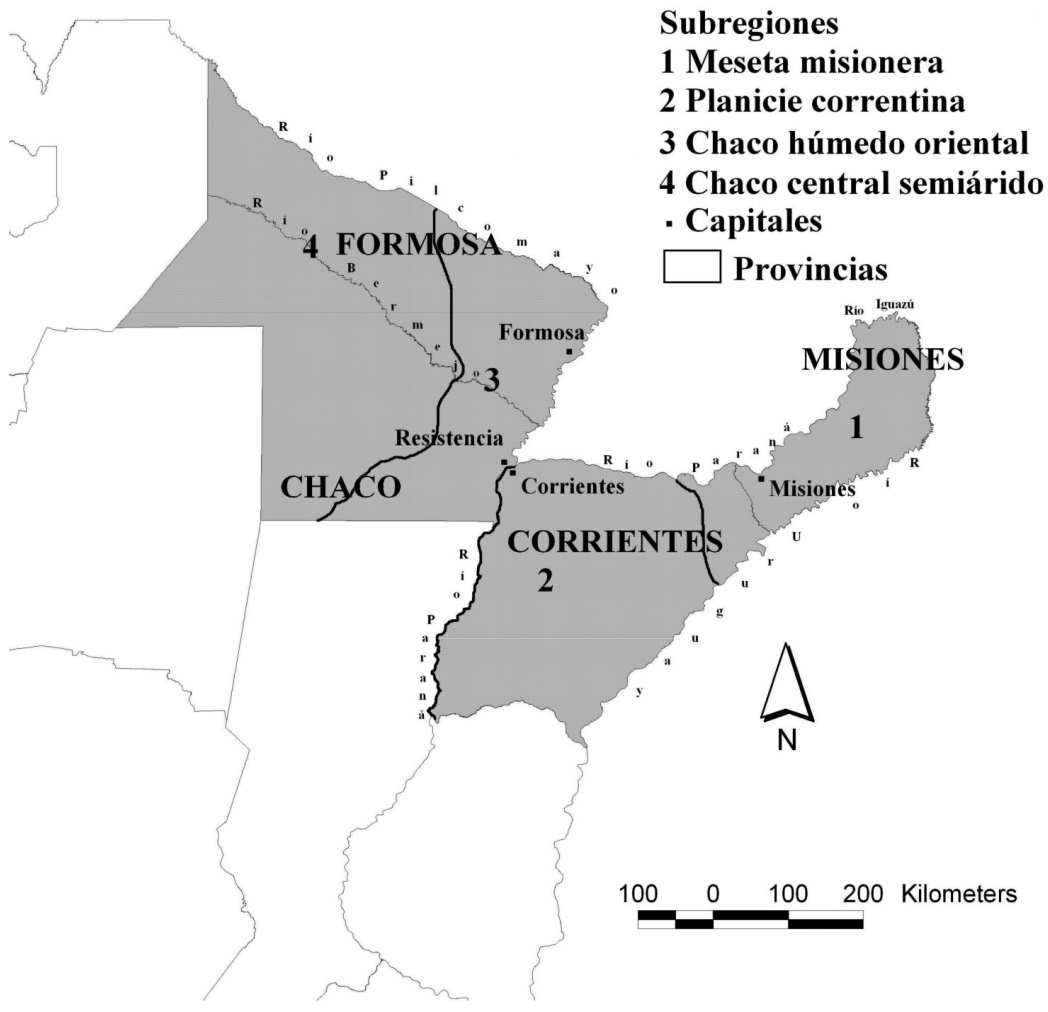

Fuente: elaboración personal.

base de su desarrollo económico. Su riqueza florística, con presencia de especies maderables cotizadas, generó desde muy temprano la penetración en el territorio para la explotación forestal y de los yerbatales naturales. El paisaje agrario se organizó en relación con tres principales cultivos industriales (té, tung y yerba) que generaron, junto con la actividad forestal, la escasa base industrial de la provincia. Estas circunstancias explican, en gran medida, el proceso emigratorio y la expansión de las periferias urbanas en esta subregión.

Las malas prácticas culturales en agricultura y forestación hacen caso omiso de las acusadas pendientes, y la desmedida explotación forestal que degradó la selva original han generado cambios ambientales. Los suelos desnudos son lavados fácilmente por las abundantes lluvias, las capas de agua no completan su recarga debido a la velocidad del escurrimiento superficial y la selva degradada ha perdido diversidad y riqueza florística. 
Todo esto ha determinado que, con excepción de la superficie del Parque Nacional Iguazú y el ángulo entre el Alto Uruguay y el Pepirí Guazú en el extremo oriental de la provincia, los procesos de erosión hídrica sean notorios, y alcancen mayor gravedad en algunos sectores de los valles fluviales y la sierra central (Casas, 1998).

2) La planicie correntina muestra dos sectores: el norte, donde predomina una depresión central ocupada por el extenso sistema del Iberá. Desde ésta se asciende hasta la rivera del río Paraná, en un territorio caracterizado por sucesión de valles y, el sur, convexo, con una elevación central (meseta de Mercedes), desde la cual descienden grandes afluentes del río Paraná. Estas condiciones hacen que Corrientes se encuentre afectada por anegamiento en las partes bajas, por erosión hídrica en los espacios con mayor pendiente y por erosión eólica en las lomadas arenosas.

$\mathrm{Su}$ estructura económica se basó tradicionalmente en la ganadería extensiva, a la cual se agregaron otras actividades como el cultivo de tabaco, cítricos, arroz, forestación, té y yerba (estos dos últimos en grandes plantaciones). Esta estructura económica primaria y escasamente diversificada explica, en gran medida, la sangría demográfica que sufre esta subregión.

3) у 4) La planicie chaqueña es parte de la extensa cuenca sedimentaria del corazón de América del Sur, desde el surco del Paraguay-Paraná hasta los contrafuertes del sistema andino. Topográficamente presenta débil inclinación noroeste-sudeste. Climáticamente la diferenciación es mucho mayor, por un lado, el chaco oriental (subregión 3) presenta excesos hídricos, red fluvial autóctona y resulta similar a las subregiones ya presentadas.

Por el otro, el chaco central semiárido (subregión 4), con marcado déficit de agua se extiende hasta las estribaciones montañosas y alcanza su mayor déficit en la línea de máxima aridez, aproximadamente a los $64^{\circ}$ de longitud Oeste (Bruniard, 1979). Entre ambos puede definirse un área central de transición, signada por la variabilidad pluviométrica interanual. Estas condiciones hacen que esta subregión se vea afectada por la erosión tanto hídrica como eólica. A esto se agrega el anegamiento de extensas superficies que en periodos de sequías pronunciadas se salinizan y constituyen un problema serio que requiere tratamiento integrado y cuidadoso. Asimismo, la explotación desmedida del bosque está, necesariamente, asociada con estos procesos erosivos.

Al igual que Misiones y a diferencia de Corrientes, la ocupación de la planicie chaqueña se ha dado, a lo largo de los últimos 130 años, en un operar centrípeto, es decir, desde la periferia hacia el corazón boscoso (Meichtry y Fantín, 2001). Se generaron tres anillos de ocupación, con diferentes estructuras: las colonias perimetrales, los latifundios intermedios 
y el corazón fiscal (Bruniard, 1979). Este espacio estuvo asociado, inicialmente, con la explotación forestal y del tanino, actividad que se complementó con la ganadería sobre terrenos bajos.

El ciclo del algodón inició a principios del siglo xx, y alcanzó una profunda crisis cinco décadas después. Desde ese entonces inicia un paulatino proceso de explotación agrícola en el NEA, sobre la base del cultivo de algodón. En la actualidad se caracteriza por ser una zona donde ha llegado la explotación agrícola intensiva, en especial de la soja que, en estas zonas, requiere de importantes cantidades de agroquímicos.

En términos generales, y con base en lo enunciado con anterioridad, las provincias constitutivas de esta región presentan indicadores económicos más bajos que la media del país Instituto Nacional de Estadísticas y Censos (2003).

\section{Metodología}

La metodología de construcción de un índice presenta gran complejidad dado que deben seleccionarse variables representativas de la situación que se pretende mostrar. Dichas variables no son directamente extrapolables a otros espacios, ya que cada uno de ellos posee especificidades y dinámicas que le son propias. Por tanto, esta característica hace que la elaboración varíe según diferentes ámbitos geográficos.

Tanto en la forma de agrupar las variables como en su ponderación existe un componente subjetivo. Se trata de un procedimiento relativamente arbitrario en el que ningún método puede justificarse con totalidad porque una variable posee mayor ponderación que otra (Tanguay et al., 2009). Además, la pertenencia de las variables a una categoría superior no es definitiva ya que alguna puede pertenecer a más de una. Este tipo de agrupación es una forma de organizar mejor la información de acuerdo con la similitud existente entre las variables y la finalidad del índice.

Para los municipios de la República Argentina se han elaborado índices de calidad de vida en diferentes escalas que contemplan la dimensión ambiental y que fueron ponderados tanto con procedimientos exclusivamente matemáticos (Boroni et al., 2005; Cepeda et al., 2004; Marinelli et al., 1999), como directos (Velázquez, 2008) con resultados espacialmente similares. Por otra parte, Ramírez (2004) y Valpreda (2007) recurren a otros métodos para evaluar variables, tal es el caso del conocido como Jerarquías Analíticas, basado en comparaciones de pares de criterios (variables).

Por su parte, Velázquez et al. (2013) realiza una recopilación bibliográfica de los estudios de calidad de vida con sus respectiva dimensión ambiental para la República Argentina, asimismo, este mismo autor 
vincula un índice de calidad ambiental con las condiciones socioeconómicas de la Región Metropolitana de Buenos Aires mientras que Longui et al. (2013) lo incluye como dimensión para el estudio de las condiciones de vida en la totalidad del territorio argentino.

Entre los trabajos más recientes que recurren al uso de los sistemas de información geográfica para el estudio de la calidad de vida y la calidad ambiental se puede mencionar a Ogneva-Himmelberger et al. (2013) y Joseph et al. (2014), quienes enfatizan la importancia de esta herramienta para el análisis espacial de los resultados.

Sobre la base de lo enunciado con anterioridad, el ICA se compuso de veintitrés componentes centrales (tabla 2), desagregados en dos grandes dimensiones: recursos recreativos y problemas ambientales. Los recursos escénicos y recreativos, a su vez, pueden ser $a$ ) de base natural (30\%) o b) socialmente construidos (30\%).

Para la valoración de los recursos recreativos de base natural partimos del supuesto de que cada lugar posee un atractivo predominante (playas, relieve, parques, espejos o cursos de agua, etc.). Según su magnitud en relación con la población residente hemos valorizado el atractivo predominante en una escala de 0 a 10 puntos. Si además de este elemento distintivo existieran otros, éstos se contabilizan y se asignan puntajes adicionales de acuerdo con su calidad (siempre respecto de la población residente).

Para la valoración de los recursos recreativos socialmente construidos se parte del supuesto de que cada lugar suele poseer varios atributos, que también pueden ser valorizados en relación con la población residente. En este caso, al tratarse de recursos "reproducibles", se califica a cada uno de ellos de 0 a 10 y se les asigna un puntaje de acuerdo al promedio respectivo.

La tercera dimensión del ICA la constituyen los problemas (costos) ambientales entendidos como los datos diversos que tienden a configurar la entidad y magnitud del problema ambiental, caracterizando y midiendo su expresión, alcance geográfico, duración temporal, naturaleza e intensidad de afectación a componentes diversos del sistema ambiental (Fernández, 2000). Sin información no hay manera objetiva de construir un escenario de aprehensión científica de los problemas ni su grado de afectación ambiental.

La dimensión incluye doce posibles problemas que pueden presentarse con diferente magnitud y que afectan las condiciones de vida de la población. Como no son excluyentes entre sí, para considerar el grado de afectación se suman las incidencias estandarizadas (puntajes omega) de cada uno para establecer el respectivo subtotal. 
La mayoría de los problemas ambientales pueden ser conocidos de manera objetiva a partir de la información provista por distintos organismos municipales, provinciales y nacionales.

Como se puede observar en la tabla 1, el ICA resulta de la combinación ponderada de:

a) $30 \%$ recursos recreativos de base natural

b) $30 \%$ recursos recreativos socialmente construidos

c) $40 \%$ problemas ambientales

Por lo que:

ICA: $\left(\left(3^{*} \mathrm{RRBN}+3^{*} \mathrm{RRSC}\right)+\left(4^{*}(10-\mathrm{PA})\right)\right) / 10$

Donde:

RRBN: Recursos recreativos de base natural ( $\Sigma$ playas, balnearios, relieve, etc.).

RRSC: Recursos recreativos socialmente construidos (x de estética, centros deportivos, etc.).

PA: Problemas ambientales ( $\Sigma$ de problemas ambientales).

El Índice de Calidad Ambiental implica una propuesta, pero también un proceso abierto y participativo, dado que en su constitución coexisten variables objetivas y subjetivas. Para las objetivas se recurrió a fuentes estadísticas mientras que, para las subjetivas, la valoración es personal, basada en información de los sitios de los municipios, fotografías, videos, viajes a los lugares analizados y bibliografía. La internet es, sin duda, el principal medio que permite recabar datos para esta finalidad. De manera recíproca, la tabla con todas las variables se encuentra disponible en la red. ${ }^{3}$

Para plasmar la dimensión espacial del índice se utilizó el ArcGis 9.2 con el método de cortes naturales para delimitar los intervalos de los indicadores. Este es el método de clasificación por defecto del programa que utiliza un algoritmo de optimización que da lugar a clases de valores similares, separados por puntos de interrupción. Es el recomendado para datos que no se distribuyen uniformemente y que no están muy sesgados hacia un extremo de la distribución.

\footnotetext{
${ }^{3}$ La tabla y la explicación y relevancia de cada una de las variables se encuentra disponible en www.cig.org.ar
} 


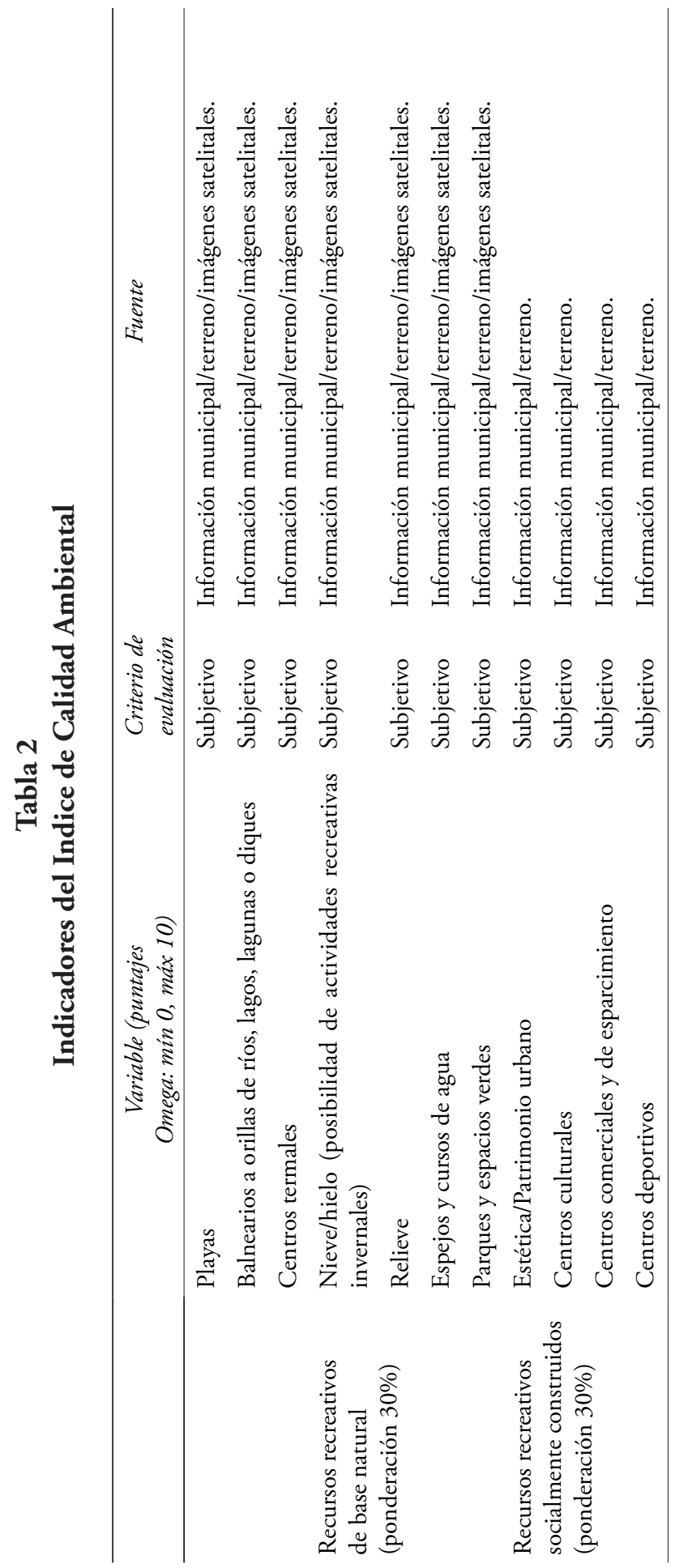




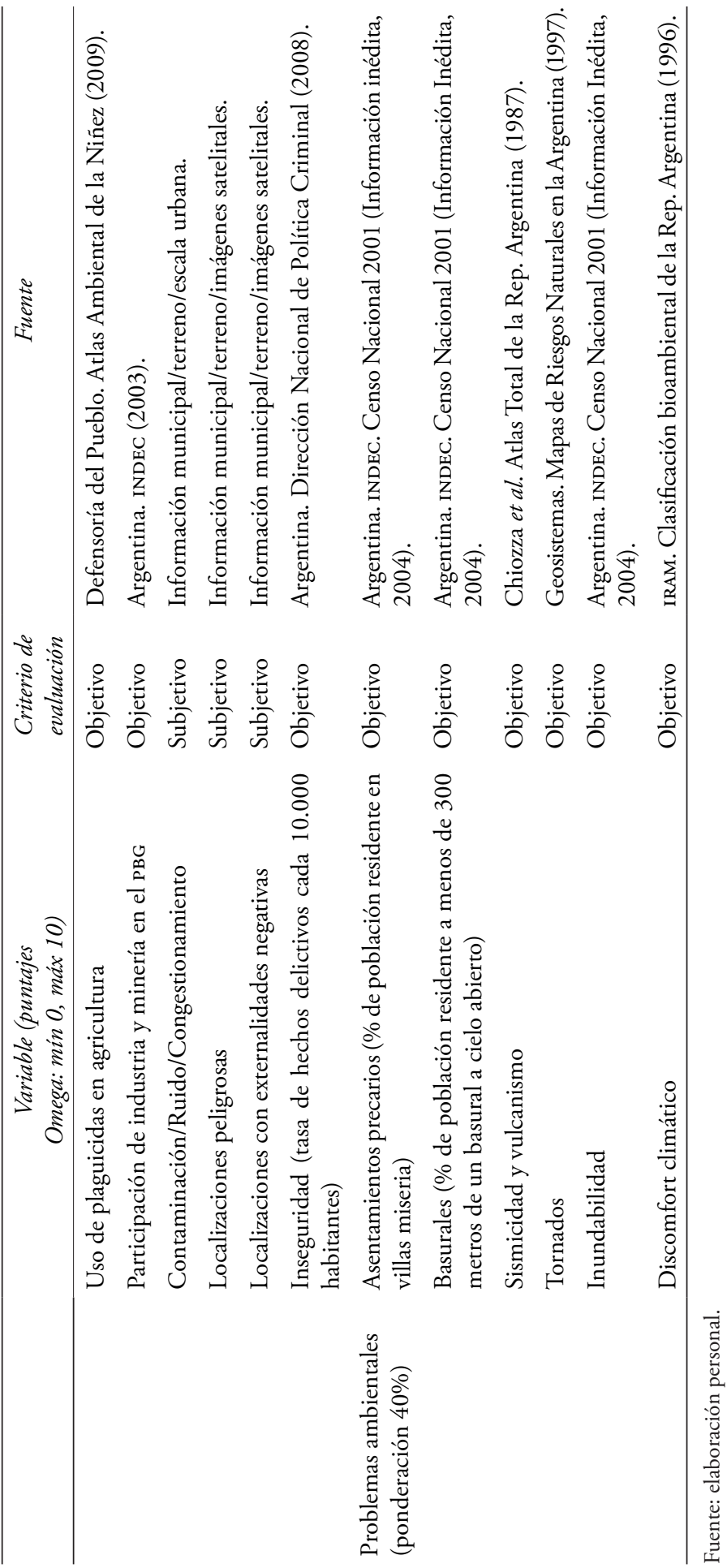




\section{Mapa II}

Recursos recreativos de base natural. Región del NEA

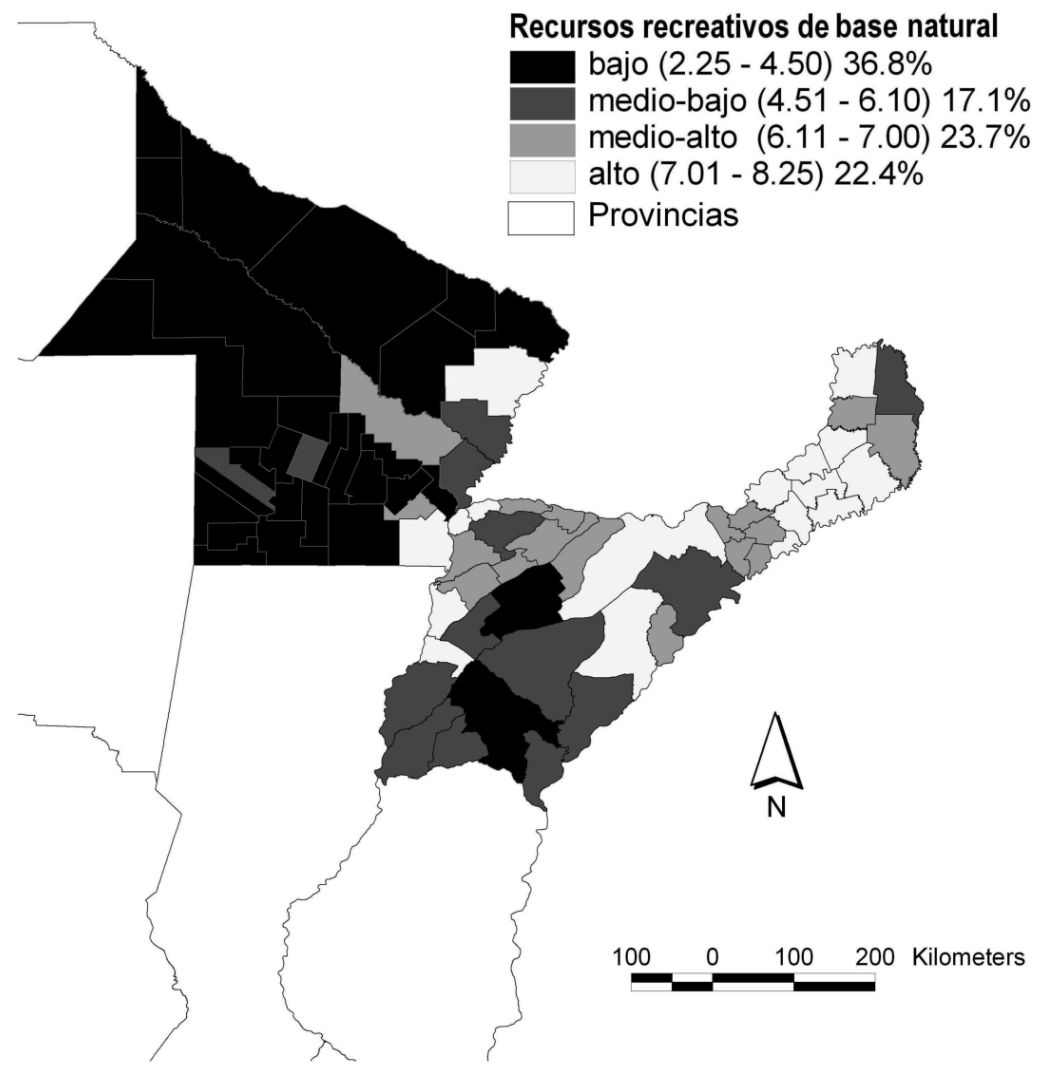

Fuente: elaboración personal.

\section{Resultados}

\subsection{Recursos recreativos de base natural}

En el mapa II se destaca, en primer lugar, un conjunto de departamentos (22.4\% del total) que cuenta con la mayor presencia de estos recursos (7.01 a 8.25 puntos). Este grupo comprende: a) gran parte de los departamentos de Misiones, $b$ ) nordeste de Corrientes (Esteros del Iberá), $c$ ) ciudades de Corrientes-Resistencia, $d$ ) dos departamentos de Corrientes sobre el río Paraná y, e) capital de Formosa. En los departamentos misioneros se conjugan relieve, exuberante vegetación y numerosos cursos de agua, muchos de los cuales cuentan con balnearios. 
Cabe destacar que en Iguazú se encuentran las Cataratas homónimas, recientemente propuestas como una de las siete nuevas "maravillas naturales". En el nordeste correntino el elemento más destacado lo constituyen los Esteros del Iberá, ecosistema que se destaca por su flora, fauna y reserva de agua dulce debidamente resguardados como parque provincial. La ciudad de Corrientes (y en menor medida Resistencia) y el contiguo departamento de San Cosme (centro turístico regional) poseen, como recurso recreativo excluyente, la costa del Paraná. Del lado correntino la barranca permite disfrutar más esta rivera, destacándose la costanera, playas y espacios verdes. Dos departamentos correntinos (Bella Vista y Lavalle) se destacan, asimismo, por sus playas de arena y balnearios sobre el Paraná. Finalmente, la capital de Formosa cuenta con atractivas costaneras, complementadas con abundante vegetación, espacios verdes y balnearios.

Este primer conjunto es el que cuenta con los mejores recursos recreativos de base natural del nordeste Argentino, lo que genera un entorno atractivo y diverso para el esparcimiento cotidiano de su población.

En segundo término, $23.7 \%$ de los departamentos del NEA, también poseen recursos recreativos atractivos, pero estos no resultan tan significativos o se encuentran más alejados de la población residente, y alcanza por tanto menor puntaje (6.11 a 7.00 puntos). En este grupo tenemos: a) diversos departamentos de Misiones y su prolongación sobre Corrientes, $b$ ) triángulo nordeste de Corrientes y c) dos departamentos chaqueńos. Los departamentos misioneros y su continuación sobre Corrientes cuentan, en general, con atractivos vinculados con la vegetación, relieve y cursos de agua. En el caso del triángulo nordeste de Corrientes, la presencia de vegetación, espacios verdes y cursos de agua compone, también, un entorno atractivo para sus habitantes. Lo mismo ocurre con los departamentos situados en el oriente chaqueño.

El tercer grupo (4.51 a 6.10 puntos) abarca $17.1 \%$ de los departamentos. Comprende varias unidades de Corrientes, el sur de la costa de los ríos Paraná y Uruguay, alrededores de dos capitales provinciales (Resistencia y Formosa) y algunos sectores del interior chaqueño. Este agrupamiento contiene menor dotación de recursos recreativos y se encuentra más alejado de los que resultan destacables. En general la puntuación resultante se basa en elementos menores, tales como parques, espacios verdes, pequeños balnearios o la contemplación del horizonte.

Finalmente, el territorio más carente de recursos recreativos (2.25 a 4.50 puntos) reúne a un significativo $36.8 \%$ de los departamentos. Se incluyen aquí a) vastos sectores del interior chaqueño y b) dos departamentos del interior correntino. El interior chaqueño constituye un ambiente semiplano, con escasa vegetación (degradada y variable de acuerdo 
con los ciclos hidrológicos) y exiguos cursos de agua, en general y alóctonos. En los pocos casos en los que el volumen de agua lo permite, las actividades recreativas se ven limitadas por la abundante carga de sedimentos y la presencia de peces carnívoros.

El clima tórrido dificulta la realización de actividades recreativas (y productivas) durante buena parte del año. Asimismo las distancias son considerables, de manera que se incrementa la sensación de monotonía. En los dos departamentos correntinos incluidos en este grupo la situación es casi similar, ligeramente atenuada por la presencia de algunos cursos de agua.

\subsection{Recursos recreativos socialmente construidos}

El mapa III exhibe la valoración de estos recursos en la región NEA. ${ }^{4}$ En primer lugar (5.76 a 8.31 puntos, tan sólo $9.3 \%$ de los departamentos) se encuentran las áreas centrales de las capitales provinciales (CorrientesResistencia, Posadas y Formosa) que, por su escala urbana, brindan servicios de especial jerarquía. Estos tres puntos constituyen los epicentros del esparcimiento regional ya que concentran las principales actividades culturales, deportivas y comerciales de sus respectivas provincias. Este grupo abarca también a las ciudades de Puerto Iguazú ubicada a la vera del río homónimo (de proyección internacional por las Cataratas) y a las segundas mayores ciudades de Misiones (Oberá) y Chaco (Presidente Roque Sáenz Peña).

En segundo término (4.63 a 5.75 puntos) se presenta un numeroso grupo de departamentos (44\%) que rodean a las ciudades mencionadas en el párrafo anterior y extensas áreas de Misiones, Corrientes y, en menor medida, Chaco. En todos los casos se trata de departamentos encabezados por localidades menores a los 50 mil habitantes, que poseen razonable dotación de servicios (educativos, sanitarios, comerciales), pero no grandes atractivos socialmente construidos, que sean destinados al esparcimiento cotidiano de su población.

Un tercer escalón (3.26 a 4.62 puntos) reúne a 33.3\% de los departamentos, bastante carentes aún en lo que respecta a amenidades socialmente construidas. Se encuentran alejados de los centros atractivos y sus cabeceras suelen ser pueblos por debajo de los 20 mil habitantes. Cuentan, tan sólo, con servicios (educativos, sanitarios, comerciales) básicos, que son menos significativos aún los destinados a la recreación.

\footnotetext{
${ }^{4}$ Recordemos que en este caso los puntajes se otorgaron sobre la base del promedio de cada uno de los recursos recreativos socialmente construidos.
} 
Finalmente en el conjunto más desprovisto de estos recursos $(1.50 \mathrm{a}$ 3.25 puntos) encontramos: a) extensas áreas del oriente formoseńo, $b$ ) cuatro departamentos relativamente cercanos a la capital chaqueña y $c$ ) dos municipios correntinos. En todos los casos se trata de unidades departamentales cuyas cabeceras están constituidas por pueblos muy pequeños, en los cuales reina la monotonía y se caracterizan por ser centros emisores de juventud.

\section{Mapa III}

Recursos recreativos socialmente construidos. Región del NEA

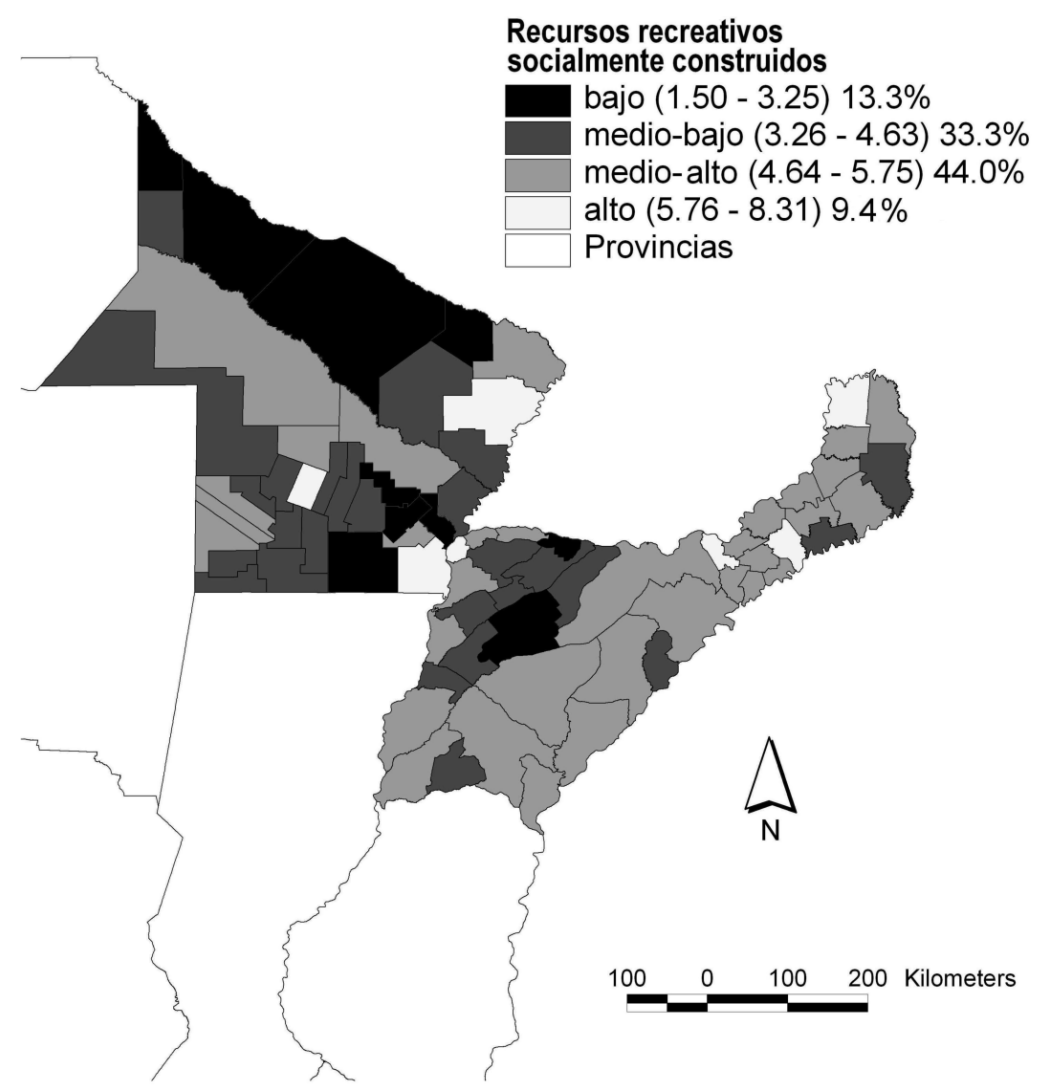

Fuente: elaboración personal.

\subsection{Problemas ambientales}

La región del NEA padece costos ambientales de diversa magnitud. Para dimensionarlos hemos propuesto diversos grados de afectación (mapa Iv). Los mayores problemas ( 2.14 a 2.87 puntos) abarcan a $10.7 \%$ de los 


\section{Mapa IV \\ Problemas ambientales. Promedio. Región del NEA}

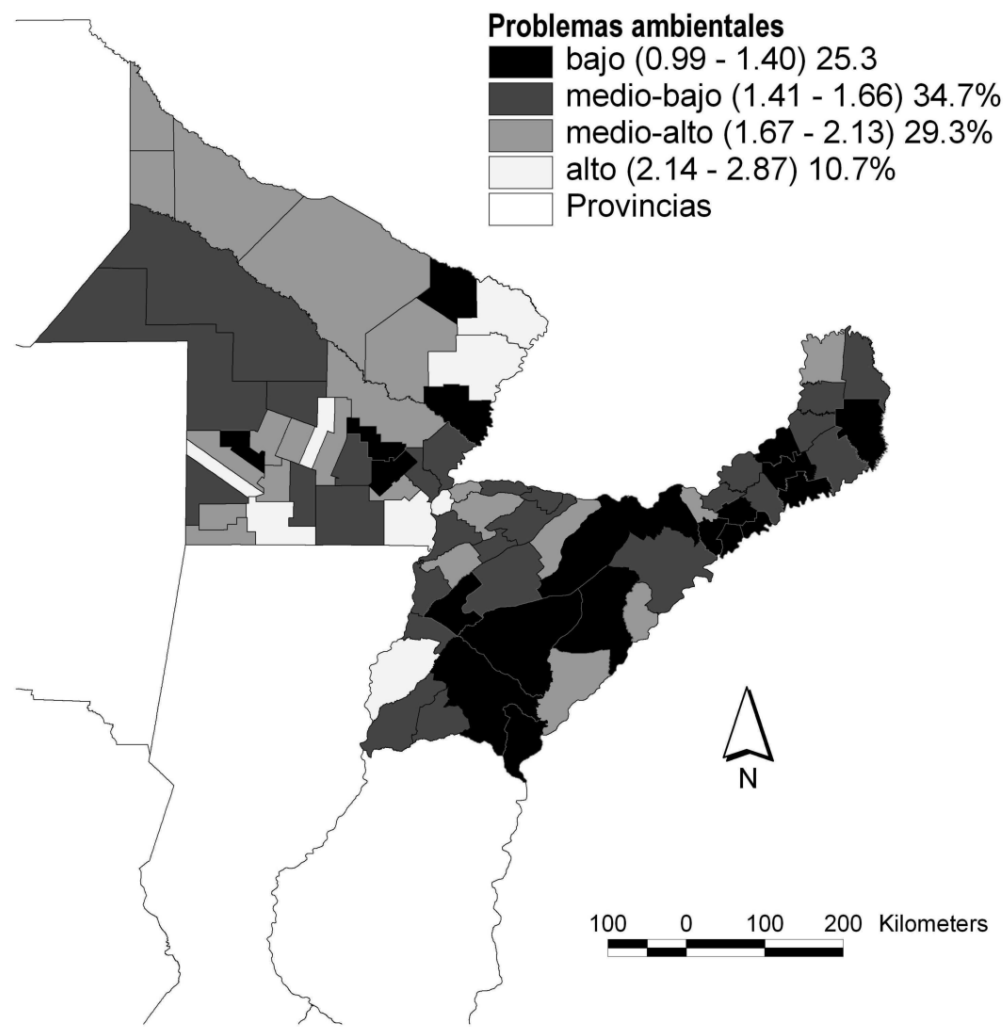

Fuente: elaboración personal.

departamentos y se presentan: a) en la periferia de Corrientes-Resistencia, b) capital de Formosa y Clorinda y c) Goya, en Corrientes. En los casos a) y b) se combinan negativamente la presencia de asentamientos precarios, inundabilidad, basurales espontáneos y una tasa relativamente alta de hechos delictivos; en el caso $c$ ) los problemas de inundabilidad alcanzan los mayores registros a nivel nacional, la presencia de basurales es muy alta y algunas localizaciones cuentan con externalidades negativas vinculadas con asentamientos militares que restringen la circulación.

En un nivel intermedio (1.41 a 2.13 puntos) se incluye a la gran mayoría de los departamentos del NEA (64\%), en los cuales se presenta una combinación de las siguientes situaciones: problemas vinculados con la escala urbana en la capital de Misiones; pobreza y asentamientos precarios en el interior de Misiones, en algunos casos coincide con altas tasas de hechos delictivos; en vastos sectores de Corrientes se presenta la misma 
situación acompañada, en algunos casos, con problemas de inundabilidad; finalmente en el interior del Chaco y, muy particularmente en el de Formosa, se observan diversas dimensiones de la pobreza y basurales espontáneos y degradación ambiental en un marco de alto discomfort climáico ${ }^{5}$, propias de su ubicación geográfica (Instituto Argentino de Normalización y Certificación, 196).

Según la Secretaría de Medio Ambiente de la República Argentina, el principal problema ambiental radica en la degradación de suelos, en particular en las zonas con ecosistemas lábiles (noroeste y suroeste del Chaco), donde existen prácticas de cultivos con tecnología no apropiada para el ambiente (Adámoli, et al., 2004).

Por último, en el grupo con menores problemas ( 0.99 a 1.40 puntos) están $25.3 \%$ del total de los departamentos que, en general, carecen de asentamientos importantes y no presentan mayores conflictos ambientales. Es la situación predominante en el interior correntino y en varios departamentos misioneros. Por el contrario, en Chaco y Formosa hay relativamente escasas áreas que presenten esta situación.

\subsection{Indice de Calidad Ambiental}

El mapa $v$ muestra que la mejor situación en lo que respecta a la calidad ambiental (7.14 a 7.72 puntos) se presenta en: $a$ ) diversos departamentos de Misiones; $b$ ) dos departamentos del noreste de Corrientes y $c$ ) en las cuatro capitales provinciales y sus alrededores. En el primer caso, la disponibilidad de recursos recreativos de base natural (relieve, espacios verdes, cursos de agua, vegetación frondosa) no se ve contrarrestada por mayores problemas ambientales (en términos relativos, ya que también los padecen). La segunda agrupación también presenta una explicación similar: los recursos escénicos están relacionados con los grandes ríos, espejos de agua y marco natural privilegiado de los Esteros del Iberá. Finalmente, las cuatro capitales provinciales quedan incluidas en este grupo fundamentalmente por sus recursos recreativos socialmente construidos, ya que las cuatro, (y muy especialmente Corrientes-Resistencia) constituyen los epicentros del esparcimiento regional.

Las dos agrupaciones siguientes (5.70 a 7.13 puntos) predominan ampliamente (53.3\% de los departamentos). Se corresponden con sitios de atractivos intermedios (fundamentalmente de base natural y, en menor

\footnotetext{
${ }^{5}$ Se entiende por comfort climático la existencia de combinaciones de parámetros ambientales (fundamentalmente temperatura, humedad, radiación y viento) que no generen estrés en el cuerpo humano. Los límites del confort climático son muy estrechos: se reducen a un abanico de temperaturas entre los $20^{\circ} \mathrm{C}$ y los $25^{\circ} \mathrm{C}$ y un rango de humedad relativa entre $30 \%$ y $70 \%$, aproximadamente (Pérez-Cueva, 2001). Fuera de estos parámetros nos encontramos frente a una situación de discomfort.
} 


\section{Mapa V \\ Índice de Calidad Ambiental. Región del NEA}

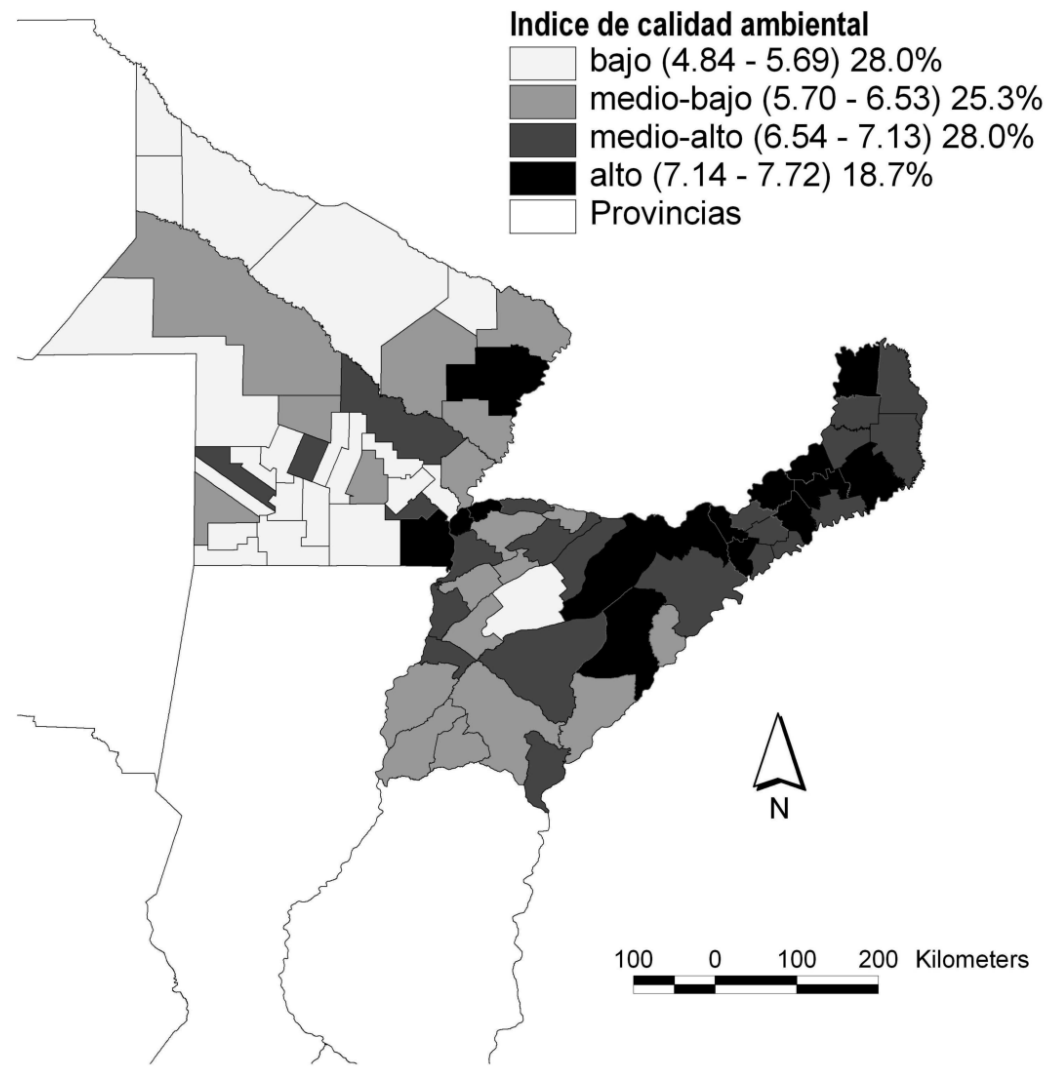

Fuente: elaboración personal.

medida, socialmente construidos) y que también padecen algunos problemas ambientales. Es la situación predominante en gran parte de los territorios misionero y correntino.

Finalmente, la peor situación relativa (4.84 a 5.69 puntos) se registra en los departamentos más carentes de recursos recreativos (tanto de base natural como socialmente construidos). Estas unidades, en algunos casos, también pueden padecer algunos problemas ambientales y constituyen $28 \%$ del área de estudio. Las peores situaciones se observan mayoritariamente en el interior chaqueño y formoseño y en un departamento del interior correntino. Misiones no registra ningún departamento en esta situación. En todos estos contextos, con muy baja calidad ambiental, la vida cotidiana suele resultar difícil y monótona. En algunos casos, también están expuestos a problemas ambientales, particularmente a periódicos 
déficit hídricos que impiden realizar cultivos y abastecer de agua potable a la población.

\section{Conclusiones}

El análisis de las condiciones de vida de la población desde una perspectiva geográfica requiere de la construcción de indicadores socioeconómicos y ambientales. A partir de la combinación de 23 variables referidas a los recursos recreativos de base natural, recursos recreativos socialmente construidos y problemas ambientales, se ha establecido un índice de calidad ambiental que muestra alto grado de diferenciación al interior de la región del nordeste Argentino (NEA). Este indicador pretende ser una síntesis, tanto de las posibilidades de esparcimiento cotidiano de la población como de los problemas ambientales que se padecen.

Todo el norte de la República Argentina, del cual forma parte el NEA, se caracteriza por presentar los mayores indicadores de pobreza y postergación del país, en especial, en los sectores más aislados de cada una de las provincias.

Los recursos recreativos socialmente construidos tienen en la región cierta lógica de "mercado", ya que se corresponden con las zonas más pobladas y, muy particularmente, donde residen los estratos con mayor capacidad de consumo. Esta característica hace que exista infraestructura asociada a la cultura o al deporte, pero que no esté al alcance de todos, ya que, por un lado, generalmente es necesario pagar por ella y, por el otro, su localización suele coincidir con la de los sectores más solventes.

Respecto de los recursos recreativos de base natural, su distribución y apropiación también resulta diferencial. A escala intra-departamental (o intra-urbana) los grupos con mayores ingresos también logran apropiarse de los mejores entornos y externalizar los "costos del desarrollo" al resto de la sociedad. De esta manera, para poder acceder alguna de las bellezas naturales es necesario pagar, excluyendo así de su utilización a ciertos sectores de la sociedad que, a su vez, son los mismos que suelen verse más afectados por los problemas ambientales.

Los resultados obtenidos muestran que sólo $18.7 \%$ de los departamentos del NEA exhibe las mejores condiciones ambientales. Este grupo comprende a diversos departamentos de Misiones; dos departamentos del noreste de Corrientes y las cuatro capitales provinciales y sus alrededores. Los factores comunes que influyen en esta valoración son la disponibilidad de atractivos naturales (relieve, cursos y espejos de agua, espacios verdes destacables) en escalas urbanas intermedias y con relativamente escasos problemas ambientales. 
Por el contrario, 28\% de los departamentos del NEA padecen condiciones adversas por la combinación de escasos atractivos (tanto naturales como socialmente construidos) y, en menor medida, problemas ambientales. Son los casos del interior chaqueño y formoseño y un departamento del interior correntino. En estos contextos, la vida cotidiana suele resultar extremadamente monótona y sujeta a gran vulnerabilidad ambiental que favorece la emigración.

Finalmente, el resto de los partidos del NEA (53.3\%) exhibe condiciones intermedias. En general esta particularidad es más producto de sus atractivos relativamente escasos (fundamentalmente de base natural) que de los problemas ambientales que padecen. Es la situación predominante en gran parte de los territorios misionero y correntino.

En el NEA se observa una clara diferenciación entre las capitales provinciales, centros indiscutidos de los recursos recreativos socialmente construidos y que carecen de problemas ambientales de relevancia debido al bajo grado de industrialización de este sector del país, donde el empleo público es una de las principales fuentes laborales; mientras que en el interior chaqueńo y formoseño la vida cotidiana suele resultar extremadamente monótona, sin atractivos naturales ni infraestructura destacable y sujeta a gran vulnerabilidad ambiental y éxodo demográfico.

En contraposición, el norte de la provincia de Corrientes y en casi la totalidad de Misiones poseen factores comunes que influyen en la valoración final del índice en cuestión: amplia y variada disponibilidad de atractivos naturales (relieve, cursos y espejos de agua, espacios verdes destacables) en escalas urbanas predominantemente intermedias y con relativamente escasos problemas ambientales.

La elaboración y análisis espacial del índice con su correspondiente cartografía constituye un aporte para la sistematización, medición y síntesis del proceso de diferenciación espacial socioambiental presente en la región del NEA de la República Argentina. Dado que el índice propuesto es el primero de índole exclusivamente ambiental para el área de estudio, en el futuro será necesario complementarlo con otros en los que predominen indicadores sociales, económicos y educativos, de manera que se pueda obtener una visión más general del bienestar de la población.

Los mapas presentados constituyen un esfuerzo de sistematización, medición y síntesis de este proceso de diferenciación. En este sentido, esperamos que además de los resultados académicos obtenidos, también puedan suministrar elementos útiles para la adecuada gestión del territorio analizado, particularmente en lo que respecta a la mitigación de los problemas ambientales y a la inversión y gestión pública de los servicios recreativos socialmente construidos. 
Luego de la aplicación en regiones particulares de la Argentina, resta para el futuro el perfeccionamiento y extensión del Índice de Calidad Ambiental al resto del territorio, revisando y actualizando sus variables constituyentes. El seguimiento y desarrollo del índice propuesto debe ser acompañado con el monitoreo de otros indicadores que permitan evaluar su desempeño a lo largo de un periodo determinado. A medida que el índice siga siendo perfeccionado y empleado en otras partes del país se podrán realizar comparaciones interregionales que permitirán obtener una visión más completa de la realidad ambiental cotidiana de todos los habitantes, dimensión insoslayable a la hora de evaluar e intervenir activamente en el bienestar de la población.

\section{Bibliografía}

Adámoli, Jorge, Sebastián Torrilla, Rubén Ginzburg (2004), Diagnóstico ambiental del Chaco Argentino, Secretaría de Ambiente y Desarrollo Sustentable, Buenos Aires.

Araña, Jorge, Carmelo León y Matías González (2003), "Valoración múltiple de bienes públicos urbanos mediante técnicas de preferencias declaradas", ponencia presentada en Hacienda Pública y Convergencia Europea X Encuentro de Economía Pública, 6-7 de febrero, Santa Cruz de Tenerife.

Boroni, Gustavo, Sebastián Gómez-Lende y Guillermo Velázquez (2005), "Geografía, calidad de vida y entropía. Aportes de la teoría de la información para la construcción de un índice de calidad de vida a escala departamental (1991-2001)" en Guillermo Velázquez y Sebastián Gómez-Lende (comps.), Desigualdad y calidad de vida en la Argentina (1991-2001). Aportes empiricos y metodológicos, Editorial REUN, Tandil, pp. 63-86.

Brulle, Robert y David Pellow (2006), "Environmental justice: Human health and environmental inequalities", The Annual Review of Public Health, vol. 27, Nonprofit Scientific Publisher, California, pp. 103-124.

Bruniard, Enrique (1979), "El Gran Chaco Argentino (ensayo de interpretación geográfica)", Geográfica, núm. 4, Universidad Nacional del Nordeste, Chaco, Argentina, pp. 2-11. 
Bruniard, Enrique y Alfredo Bolsi (1992), "Región agro-silvo-ganadera con frentes pioneros de ocupación del Nordeste", en Juan Roccatagliata (coord.), La Argentina. Geografía general y los marcos regionales, Planeta, Buenos Aires, pp. 529-577.

Casas, Roberto (1998), "Los procesos de degradación y la conservación de suelos en la República Argentina”, en Diana Durán (comp.), La Argentina ambiental. Naturaleza y sociedad, Editorial Lugar, Buenos Aires, pp. 143-178.

Catalán-Vázquez, Minerva y Jarillo-Soto, Edgar (2010), "Paradigmas de investigación aplicados al estudio de la percepción pública de la contaminación del aire", Revista Internacional de Contaminación Ambiental, 26 (2), unam, México, pp. 165-178.

Cepeda, Rosana, Claudia Marinelli, Sebastián Gómez-Lende y Guillermo Velázquez (2004), “Técnicas de análisis multivariado para la determinación de calidad de vida", en Memorias del Primer Seminario Argentino de Geografía Cuantitativa, 26-27 de agosto, Buenos Aires.

Chiozza, Elena, Ricardo Figueira, y Alicia Iglesias, (1987), Atlas total de la República Argentina, Centro Editor de América Latina, Buenos Aires.

Defensoría del Pueblo de la Nación (2009), Atlas del riesgo ambiental de la niñez, Unicef-ops, Buenos Aires.

Dirección Nacional de Política Criminal (2008), Estadísticas en materia de criminalidad. Ministerio de justicia, seguridad y derechos humanos, Buenos Aires, <www.jus.gov.ar/areas-tematicas/estadisticasen-materia-de-criminalidad.aspx>, 8 de agosto de 2010.

Escobar, Luis (2006), "Indicadores sintéticos de calidad ambiental: un modelo general para grandes zonas urbanas", EURE, XXXII (96), Santiago de Chile, pp. 73-98.

Fernández, Roberto (2000), La ciudad verde: teoría de la gestión ambiental urbana, Espacio Editorial, Buenos Aires.

Gallopin, Gilberto (1996), "Environmental and sustainability indicators and the concept of situational indicators as acost-effective ap- 
proach", Environmental Modelling and Assessment, 1 (3), Centro Internacional de Agricultura Tropical, Cali, pp. 101-117.

Gallopin, Gilberto (2006), Los indicadores de desarrollo sostenible. Aspectos conceptuales y metodológicos, Fodepal, Santiago de Chile.

Geosistemas (1997), Mapa de riesgos naturales en la Argentina, Geosistemas, Buenos Aires.

Herzer, Hilda y Raquel Gurevich (1996), "Degradación y desastres: parecidos y diferentes: tres casos para pensar y algunas dudas para plantear”, en María Fernández (comp.), Ciudades en riesgo. Degradación ambiental, riesgos humanos y desastres, Red de Estudios Sociales en Prevención de Desastres en América Latina, Lima, pp. 75-91.

IRAM (Instituto Argentino de Normalización y Certificación) (1996), Clasificación bioambiental de la República Argentina, IRAM, Buenos Aires.

INDEC (Instituto Nacional de Estadísticas y Censos) (2003), PBG por provincia y sector de actividad económica, Buenos Aires, <www. mecon.gov.ar/secpro/dir_cn/default1.htm>, 13 de Abril de 2010.

INDEC (Instituto Nacional de Estadísticas y Censos) (2010), Censo 2010. Total del país. Población por sexo, tasa de variación intercensal, según provincia. Años 2001-2010, <http://www.censo2010.indec.gob.ar/ preliminares/cuadro_totalpais.asp>, 13 de Abril de 2013.

Joseph, Myrtho, Wang Fahui y Wang Lei (2014), "Grs-based assessment of urban environmental quality in Port-au-Prince, Haiti”, Habitat International, vol. 41, Elsevier, London, pp. 33-40.

Longui, Fernando, Pablo Paolasso, Alfredo Bolsi, Guillermo Velázquez y Juan Pablo Celemín (2013), "Fragmentación socioterritorial y condiciones de vida en la Argentina en los albores del siglo xxı", Revista Latinoamericana de Población, 7 (12), Asociación Latinoamericana de Población, Buenos Aires, pp. 99-131.

Luengo, Gerardo (1998), "Elementos para la definición y evaluación de la calidad ambiental urbana. Una propuesta teórico-metodológica", ponencia presentada en el IV Seminario Latinoamericano de Calidad de Vida Urbana, 8-11 de septiembre, Tandil. 
Marinelli, Claudia, Sebastián Torcida, Rosana Cepeda, María García y Guillermo Velázquez (1999), "Un procedimiento alternativo para la selección estadística de variables de calidad de vida”, en Guillermo Velázquez y María García (comps.), Calidad de vida urbana: aportes para su estudio en Latinoamérica, Centro de Investigaciones Geográficas-unCPBA, Tandil, pp. 133-142.

McCann, Eugene (2004), “'Best places': Interurban competition, quality of life and popular media discourse”, Urban Studies, 41 (10), Sage publications, London, pp. 1909-1929.

Meichtry, Norma y Alejandra Fantín (2001), “Condiciones ambientales, procesos de ocupación y desarrollo y calidad de vida en el nordeste de Argentina”, en Guillermo Velázquez, Geografia, calidad de vida y fragmentación en la Argentina de los noventa, Universidad Nacional del Centro de la Provincia de Buenos Aires-Centro de Investigaciones Geográficas, Tandil, pp. 114-131.

Meichtry, Norma y Alejandra Fantín (2008), “Territorios en regresión. Calidad de vida y pobreza en el nordeste argentino", en Guillermo Velázquez, Geografía y Bienestar, Eudeba, Buenos Aires, pp. 299-321.

Metzger, Pascale (2006), "Medio ambiente urbano y riesgos: elementos de reflexión”, en María A. Fernández (comp.), Ciudades en riesgo. Degradación ambiental, riesgos humanos y desastres, Red de Estudios Sociales en Prevención de Desastres en América Latina, Lima, pp. 61-77.

Ogneva-Himmelberger, Rahul Rakshit Yelena y Pearsall Hamil (2013), "Examining the impact of environmental factors on quality of life across Massachusetts", The Professional Geographer, 65 (2), American Society of Professional Geographers, Washington, pp. 187-204.

Pérez-Cueva, Alejandro (2001), "Clima y confort en las ciudades: la ciudad de Valencia”, Métode, núm. 31, Universitat de València, Valencia, pp. 147-150.

Pieri, Christian, Julian Dumanski, Ann Hamblin y Anthony Young (1995), "Land quality indicators", en World Bank Discussion Papers, The World Bank, Washington, pp. 63-315. 
Popolizio, Eliseo (1963), Un problema de geomorfología aplicada en la provincia de Corrientes, Universidad Nacional del Litoral, Rosario.

Ramírez, Mirta (2004), "El método de jerarquías analíticas de Saaty en la ponderación de variables. Aplicación al nivel de mortalidad y morbilidad en la provincia del Chaco", Comunicaciones Cientifcas y Tecnológicas, Universidad Nacional del Nordeste, Resistencia, pp. 1-4.

Riera, Pere (1994), Manual de valoración contingente, Instituto de Estudios Fiscales, Madrid.

Rogerson, Robert (1999), "Quality of life and city competitiveness”, Urban Studies, 36 (5-6), Sage publications, London, pp. 969-985.

Rotmans, Jan (1997), "Indicators for sustainable development", en Jan Rotmans y Bert de Vries (eds.), Perspectives on Global Change: The TARGETS approach, Cambridge University Press, Cambridge, pp. 187-204.

Santos, Milton (1996), A natureza do espaço. Técnica e tempo. Razâo e emoçâo, Hucitec, Sâo Paulo.

Sterimberg, Edith, Carlos Sánchez, Adriana Cuervo de Forero y Juan Ramírez (2004), Diseño de un sistema de indicadores socioambientales para el distrito capital de Bogotá, CEPAL-Naciones Unidas, Santiago de Chile.

Szas, Andrew y Michael Meuser (1997), "Environmental inequalities: Literature review and proposals for new directions in research and theory", Current Sociology, 45 (3), Sage Journals, London, pp. 99-120.

Tanguay, Georges, Juste Rajaonson, Jean Lefebvre y Paul Lanoie (2009), "Measuring the sustainability of cities: A survey-based analysis of the use of local indicators", Cirano, vol. 2, Université du Quebec, Montreal, pp. 1-29.

Valpreda, Claudia (2007), "Sistema de Información Geográfica (sIG)teledetección y evaluación multicriterio (EMC) en un estudio de evaluación de impacto ambiental (EIA)", en Memorias XI Con- 
ferencia Iberoamericana de Sistemas de Información Geográfica, 29-31 de mayo, Buenos Aires.

Velázquez, Guillermo (2008), Geografía y bienestar, Eudeba, Buenos Aires.

Velázquez, Guillermo, Fernando Longui, Claudia Mikkelsen y Juan Pablo Celemín (2013), "Estudios sobre geografía y calidad de vida en la Argentina. Cinco décadas de aportes bibliográficos", Hologramática, 19 (1), Universidad Nacional de Lomas de Zamora, Lomas de Zamora, pp. 77-105.

Van Kamp, Irene, Kees Leidelmeijer, Gooitske Marsmana y Augustinus de Hollander (2003), "Urban environmental quality and human well-being. Towards a conceptual framework and demarcation of concepts; a literature study", Landscape and Urban Planning, 65 (1-2), Elsevier, London, pp. 5-18.

Weston, Joe (2000), "EIA decision-making theory and screening and scoping in uk practice", Journal of Environmental Planning and Management, 43 (2), Routledge, Oxford, pp.185-203.

Wilkins, Hugh (2003), "The need for subjectivity in EIA: discourse as a tool for sustainable development", Environmental Impact Assessment Review, 23 (4), Elsevier, Ontario, pp. 401-414.

Recibido: 15 de diciembre de 2011. Reenviado: 21 de diciembre de 2011. Aceptado: 26 de septiembre de 2013.

Juan Pablo Celemín. Argentino. Doctor en geografía por la Universidad Nacional del Sur, Argentina. Master en conservación y gestión del medio natural (Universidad Internacional de Andalucía, España). Licenciado en geografía (Universidad Nacional de Mar del Plata, Argentina). Investigador Asistente del Consejo Nacional de Investigaciones Científicas y Técnicas con lugar de trabajo en el Centro de Investigaciones Geográficas de la Universidad Nacional del Centro de la Provincia de Buenos Aires, Argentina. Su línea de investigación se centran en el uso de sistemas de información geográfica en temáticas ambientales urbanas y regionales. Entre sus publicaciones destacan, en coautoría: La calidad ambiental en la Argentina. Análisis regional y departamental (c. 2010), CIG-Conicetreun-Universidad Nacional del Centro de la Provincia de Buenos Aires, 
Tandil (2013); “Calidad ambiental y nivel socioeconómico: su articulación en la Región Metropolitana de Buenos Aires", Scripta Nova, XVII (441) Universidad de Barcelona, Barcelona, pp. 1-33 (2013) y "Proposal and application of an environmental quality index for the Metropolitan Area of Buenos Aires (Argentina)", Geografisk Tidsskrift-Danish Journal of Geography, 112 (1), Taylor and Francis Group, Buenos Aires, pp. 15-26 (2012).

Guillermo Ángel Velázquez. Argentino. Doctor en ciencias sociales con orientación en geografía por la Universidad de Buenos Aires. Investigador Principal del Consejo Nacional de Investigaciones Científicas y Técnicas. Exdirector del Centro de Investigaciones Geográficas de la Universidad Nacional del Centro de la Provincia de Buenos Aires, Argentina. Ha sido profesor invitado en las universidades de Alcalá de Henares, Complutense y Valladolid (España) y Lund (Suecia). Su línea de investigación se centra en el estudio de la calidad de vida en la República Argentina en distintas escalas de análisis. Entre sus publicaciones destacan, en coautoría: La calidad ambiental en la Argentina. Análisis regional y departamental (c. 2010), Cig-Conicet-Reun-Universidad Nacional del Centro de la Provincia de Buenos Aires, Tandil (2013); "Calidad ambiental y nivel socioeconómico: su articulación en la Región Metropolitana de Buenos Aires”, Scripta Nova, XVII (441), Universidad de Barcelona, Barcelona, pp. 1-33 (2013) y "Proposal and application of an environmental quality index for the Metropolitan Area of Buenos Aires (Argentina)", Geografisk Tidsskrift-Danish Journal of Geography, 112 (1), Taylor and Francis Group, Buenos Aires, pp. 15-26 (2012). 